\title{
Incubadoras universitarias y su papel en la comercialización del conocimiento: caso Universidad Técnica de Machala
}

\section{University incubators and its role in the commercialization of knowledge: case Universidad Técnica de Machala}

John Alexander Campuzano Vásquez

Universidad Técnica de Machala, Ecuador

Samuel Efraín Chuquirima Espinoza

Universidad Técnica de Machala, Ecuador

Víctor Betancourt Gonzaga

Universidad Técnica de Machala, Ecuador

Autor para correspondencia: jcampuzano@utmachala.edu.ec, schuquirima@utmachala.edu.ec, vigonzaga@utmachala.edu.ec

Fecha de recepción: 23 de Enero de 2018 - Fecha de aceptación: 25 de julio de 2018

Resumen: Sobre la base de una exhaustiva revisión bibliográfica, este artículo recoge el estado actual de procesos de incubación en varios países y el marco referencial que deben aplicar universidades que quieren implementar una incubadora universitaria exitosa en Ecuador. Los resultados revelan que no todas las incubadoras existentes en el país cumplen con los requisitos exigentes de la Secretaría Nacional de Ciencia y Tecnología, con lo que de ochenta incubadoras existentes solo 14 tienen acreditación para incubar empresas bajo estándares de calidad, además demuestra que hay una débil presencia de universidades apoyando procesos de emprendimiento y de innovación en el país. Y en este sentido la Universidad Técnica de Machala si quiere concretar procesos fuertes de investigación aplicada debe elegir el tipo de incubadora que mejor responda a su realidad institucional.

Palabras Claves: incubación; universidad; empresa; emprendimiento

\begin{abstract}
Based on an exhaustive bibliographical review, this article gathers the current state of incubation processes in several countries and the referential framework that must apply universities that want to implement a successful university incubator in Ecuador. The results reveal that not all the existing incubators in the country meet the demanding requirements of the National Secretariat of Science and Technology, so that of only eighteen existing incubators, only 14 have accreditation to incubate companies under quality standards, in addition shows that there is a weak presence of universities supporting entrepreneurship and innovation processes in the country. And in this sense the Technical University of Machala if it wants to specify strong processes of applied research must choose the type of incubator that best responds to its institutional reality.
\end{abstract}

Key words: incubation; university; company; entrepreneurship 


\section{Introducción}

Las instituciones de educación superior (IES como se conocerá de aquí en adelante) cumplen sus misiones tradicionales (enseñanza e investigación) y además, emprenden otras nuevas que reflejan las contribuciones económicas, sociales y culturales de su evolución territorial (Trippl, Sinozic, \& Lawton Smith, 2015). Estas nuevas misiones obligan a las universidades del sistema de educación superior ecuatoriano a innovar en sus actividades sustantivas para responder eficiente y eficazmente a este proceso evolutivo.

Bikse, Lusena-Ezera, Rivza, \& Volkova (2016) mencionan ya la importancia de contar con una universidad empresarial capaz de apoyar las nuevas ideas de los estudiantes, potenciando la co-creación con socios externos, como una fuente impulsora para lograr educación superior sostenible. Un enfoque orientado a la práctica empresarial, que puede entenderse no solo desde la rentabilidad, sino en un amplio aspecto organizativo no lucrativo.

En la línea de cambio, Freire (2016) no deja de señalar que el sistema educativo ha de sentar las bases para proporcionar las capacidades y habilidades necesarias con el fin de que los jóvenes, una vez se convierten en egresados, puedan desempeñar alguno de los múltiples roles que existen en el mundo laboral actual, (una de las tareas pendientes en nuestras universidades) que como se mencionó está motivando el desarrollo de formas alternativas al cumplimiento de estos objetivos. Gozali, Masrom, \& Zagloel (2016) presentan un nuevo enfoque que apoya la idea que las universidades deben motivar a los jóvenes graduados para convertirse en empresarios de tecnología, un aspecto que cobra relevancia en las universidades latinoamericanas y en el mundo desarrollado con más fuerza, como una forma de alcanzar niveles de desarrollo más rápido.

Tres aspectos relacionados; una universidad mirando al sector empresarial, otra capacitando para la inserción laboral y una más creando tecnología con sus egresados. Se puede decir, que estas tres miradas deben ser asumidas por la universidad actual, sin desmedro de otras que surjan del análisis de la realidad territorial tal como se enunció anteriormente.

Ahora bien, para lograr el cumplimiento de objetivos innovadores de la universidad pública ecuatoriana, esta está proponiendo procesos de transformación hacia la excelencia que lleva a complementar sus misiones tradicionales (investigación y enseñanza) con una tercera, que contribuye al desarrollo y prosperidad territorial mediante un papel activo en la comercialización de sus conocimientos a través de spin-offs, patentes y licencias (Grimaldi, Kenney, Siegel, \& Wright, 2011).

Actividades que están íntimamente relacionadas con la implementación de nuevas estructuras de incentivos y recompensas, que promuevan la comercialización de la investigación científica universitaria tanto de estudiantes y docentes, que lleva a una competencia por patentes y licencias como forma de demostrar avances en la investigación desde las universidades tanto públicas como privadas y la consecución de mayores presupuestos operativos.

Con estos antecedentes, este artículo propone analizar los lineamientos que deben ser considerados para crear y tener incubadoras de empresas exitosas en universidades públicas y en provincias con débil desarrollo industrial como es el caso de la provincia de El Oro en Ecuador, 
en donde tiene presencia la Universidad Técnica de Machala la misma que por su crecimiento y categorización actual requiere implementar procesos que visibilicen su labor investigativa y vinculación con la sociedad.

\section{Desarrollo}

La idea de incubadoras de empresas surgió en los Estados Unidos de América a mediados del siglo pasado después de la Segunda Guerra Mundial como un nuevo instrumento para revivir las fábricas abandonadas y los distritos industriales, ofreciendo a los nuevos empresarios oficinas y recursos de uso compartido (Bikse et al., 2016). La primera incubadora se estableció en 1959 en Batavia, Nueva York, en los Estados Unidos (Aerts, Matthyssens, \& Vandenbempt, 2007).

A partir de los años setenta, las incubadoras de empresas se han extendido por todo el mundo (Albert y Gaynor, 2001), debido al desarrollo de sectores de servicios que crearon la necesidad de contar con micro, pequeñas y medianas empresas. Mientras tanto, en la década de 1970 el concepto se extendió a Europa cuando la siderúrgica estatal británica British Steel Corporation, ante una reestructuración masiva de la industria siderúrgica del Reino Unido, formó una subsidiaria, British Steel Industry Ltd (BSI) que fue el vehículo para encontrar nuevos usos en "espacio de trabajo gestionado" (Smith \& Zhang, 2012).

De acuerdo al recorrido histórico que presenta Theodorakopoulos, Kakabadse, \& Mcgowan (2014) las incubadoras de negocios han avanzado desde las de primera generación, aquellas comprendidas entre 1980 a 1990 caracterizadas por proporcionar espacio físico e instalaciones compartidas para aquellos que eran parte del proceso de incubación, más tarde aparecen incubadoras de segunda generación cuyo periodo va de 1991 al 2000, proporciona además de lo anterior, una variedad de servicios de apoyo, de asesoramiento empresarial y redes de trabajo.

Las incubadoras de tercera generación cuya temporalidad va desde el año 2001 hasta la presente, se caracterizan por adicionar apoyo proactivo, mentoría y coaching, aceleración de negocios y desarrollo de redes, convirtiéndose en proveedoras de servicios especializados.

China ejemplifica esta transición, autores como Chandra, He, \& Fealey (2007) señalan que las incubadoras en China han evolucionado desde la primera a la segunda generación, pasando de una incubadora integral (a saber, abierta a una variedad de incubadoras de alta tecnología, como software, bio-productos farmacéuticos y nuevos materiales) a un enfoque más especializado (es decir, concentrándose en industrias de alta tecnología).

La participación de las universidades en la incubación fue el resultado de profundos cambios institucionales en el sistema universitario que tuvieron lugar a lo largo de muchos años, no sólo en los Estados Unidos sino en todo el mundo (Smith \& Zhang, 2012). Investigadores atribuyen en el caso de los Estados Unidos al cambio de papel de la investigación pública en las universidades, que las llevan más allá de su misión de docencia y de investigación, a encontrar una posición competitiva de la manufactura estadounidense frente a la industria de Japón, además de encontrar un clima favorable a la hora de reclamar derechos legales sobre los descubrimientos realizados por el personal docente y su posterior comercialización. 
Una situación similar se vive actualmente en el Ecuador, las universidades ecuatorianas comienzan a mirar no solo su misión de docencia e investigación, sino que buscan en la extensión la posibilidad de potenciar significativamente su trabajo investigativo para comercializar y explotar descubrimientos universitarios en ciencia y tecnología. Para esto, se comienza a pensar en nuevas unidades organizativas dentro de las universidades siendo las incubadoras de empresas una de las alternativas

Hay que considerar teóricamente varias características que debe tener una Universidad Emprendedora (UE) que quiera implementar estrategias de desarrollo innovadoras, como es el caso de tener una incubadora de empresas. Por ejemplo, Arnaut (2010) ofrece las principales características de una Universidad Emprendedora desde diferentes aspectos basados en las opiniones de diferentes autores (Burton, 2003, Etzkowitz, 2004, Hannon, 2008, Robertson, 2008, Gibb, Haskins y Robertson, 2009 ).

Arnaut (2010) señala que una UE se caracteriza por una serie de factores clave, como un fuerte liderazgo que desarrolla capacidades empresariales en sus estudiantes y en el personal de su campus; fuertes lazos con sus actores externos que aportan un valor añadido; entrega de resultados empresariales con impacto en las personas y en las organizaciones; técnicas innovadoras de aprendizaje que inspiran la acción emprendedora; enfoques multidisciplinarios de la educación que imitan la experiencia del mundo real y se centran en resolver desafíos mundiales complejos; por citar los más importantes.

En este sentido, para proponer la creación de una incubadora de negocios es necesario que las IES miren hacia adentro y determinen la existencia o no de las condiciones que señala Arnaut. Por lo que una incubadora al ser un mecanismo para transferencia de tecnología puede promover el crecimiento de la innovación y el espíritu empresarial en las universidades. La incubadora se ve generalmente como un catalizador que permite el proceso de transferencia de conocimientos y la comercialización de la innovación (Wonglimpiyarat, 2016). Etzkowitz (2003) ya hacía aportes a esta transferencia y creación de conocimientos con innovación, con su modelo de Triple Hélice que considera la vinculación entre universidad, empresas y gobierno como una potente forma de generar innovación basada en el conocimiento de la sociedad.

Crear una incubadora de empresas en una universidad, no debe hacerse sin tener precisión sobre la gama completa e integrada de servicios que se pretende proporcionar como lo señala (Gozali et al., 2016). Estos investigadores afirman que para sentar las bases para un programa de incubación exitoso, los desarrolladores de incubadoras deben primero invertir tiempo y dinero en un estudio de factibilidad eficaz que ayude a determinar si el proyecto propuesto tiene un mercado sólido, una base financiera consistente y un fuerte apoyo de la comunidad, factores críticos asociados con el éxito de una incubadora.

A lo anterior, se suma la necesidad de contar con un programa modelo de incubación de empresas que tome como referencia las mejores prácticas de la industria, para lo cual será necesario considerar las potencialidades del territorio, en el caso de Ecuador hay que considerar las zonas de planificación territorial que definen incluso agendas de transformación productiva que entregan a cada provincia sus sectores estratégicos de innovación. 
Revisando a Verma (2004) en la figura 1 se observan algunos factores de éxito para una incubadora, se tiene como variable dependiente el grado de éxito de la incubadora y como factores independientes que explican la varianza de la variable dependiente, (1) servicios compartidos, (2) instalaciones y ubicación, (3) financiamiento y apoyo, (4) criterios de salida, y (5) tutoría y establecimiento o trabajo en redes.

Estos cinco factores que presenta Verman, son una guía importante para las IES que quieren implementar una incubadora, en donde no solo es necesaria la factibilidad financiera como se acostumbra pensar, sino los recursos humanos universitarios que visibilicen a especialistas por área, los servicios identificados a entregar, junto a las instalaciones necesarias para incubar empresas y los aspectos legales que se deben tener presente.

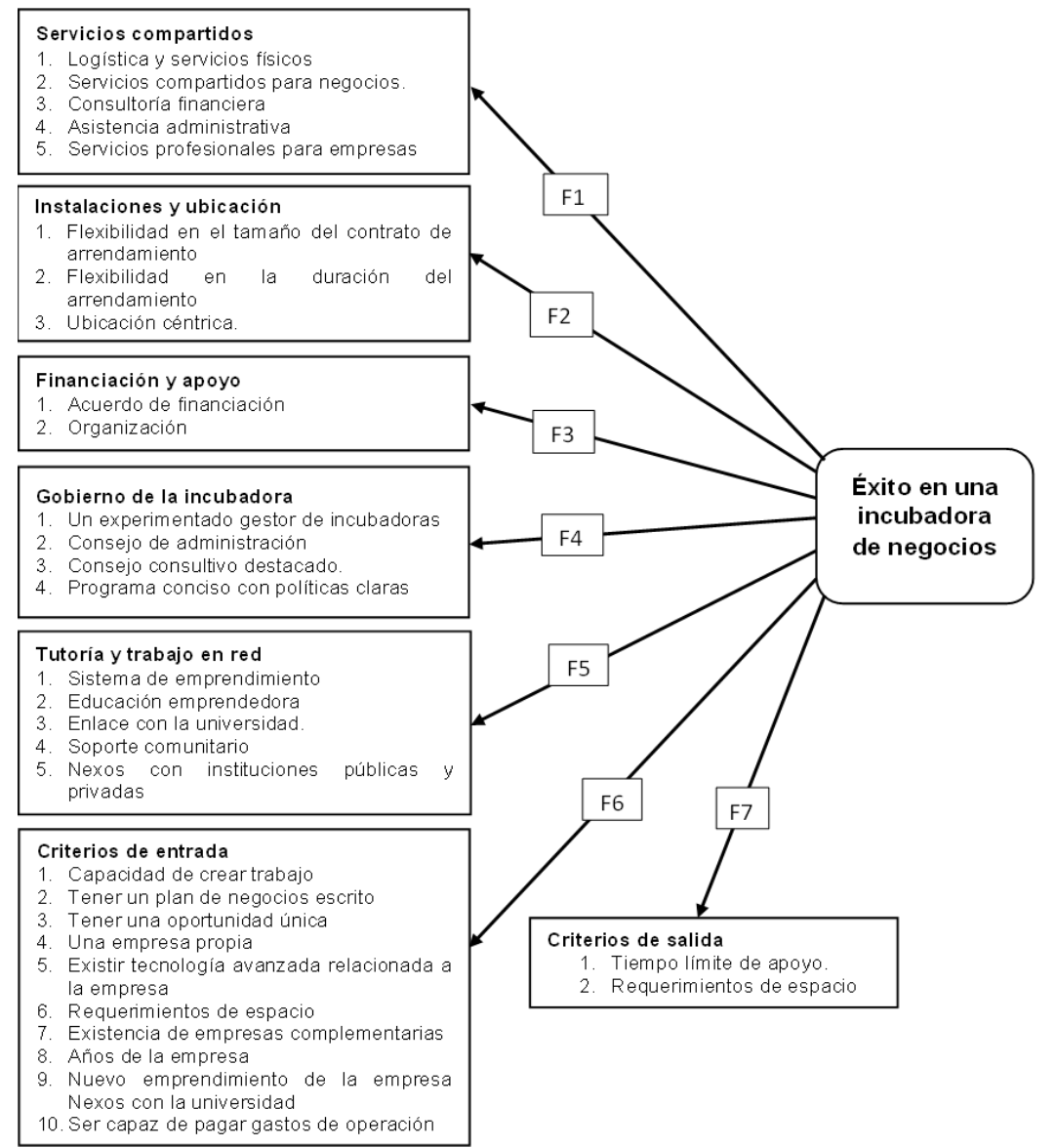

Figura 1. Factores exitosos para la incubación tomado y adaptado de Verman (2004)

Fuente: (Verman, 2004)

Elaboración de autores.

Autores como Peña-Vinces, Bravo, Álvarez, \& Pineda (2011) sugieren siete pilares o factores si se les puede llamar así, para la supervivencia de incubadora o para su éxito, y en lo referente al modelo de incubación proponen que se deberían considerar 23 aspectos:

- Portafolio de servicios

- Procesos de Coaching 
- Mecanismos de participación y acuerdos de derechos de autor

- Implementación de incubadoras virtuales

- Laboratorios de ideas y un HELP DESK

- Personalidades universitarias en la asesoría especializada

- Centro de recursos legales

- Centro de competencia y formación

- Bolsa de trabajo

- Tasa variable sobre el canon de arrendamiento

- Apoyo gerencial

- Entrenamiento y facilitación de servicios y consejo de asesores

- Soporte para el desarrollo de la comercialización

- Filtros para seleccionar a los emprendedores y empresas que desean vincularse a la incubadora

- Contratos con los incubados

- Políticas de graduación

- Tiempo óptimo de incubación

- Criterios de selección

- Normas y procedimientos de admisión

- Sistema de seguimiento y monitoreo

- Programas de capacitación con universidades y colegios

- Programas de apoyo a la internalización de empresas incubadas

- Gestión de recursos para la internacionalización y globalización de los incubados

De estos veintitrés aspectos, se considera que en el caso de una incubadora para la Universidad Técnica de Machala ubicada en la provincia de El Oro, por su tamaño mediano dentro del sistema nacional universitario, se debe dar mayor importancia al portafolio de servicios, criterios de selección, sistemas de seguimiento y monitoreo, contratos con los incubados y filtros para seleccionar a los emprendedores y empresas que desean vincularse a la incubadora, sumando el desarrollar un ecosistema emprendedor.

Además, es necesario recoger requisitos básicos que Hernández \& González (2016) señalan para que un ecosistema de emprendimiento prospere y se autoabastezca, como es el caso de que la comunidad emprendedora esté necesariamente encabezada por empresarios, especialmente aquellos que dirigen pequeñas y medianas empresas bien establecidas, ya que estos empresarios tienen más experiencia y son los verdaderos contrafuertes de un ecosistema empresarial.

Albort-Morant \& Oghazi (2016) ratifican la utilidad del asesoramiento y el apoyo de las incubadoras de empresas para empresarios e inquilinos de incubadoras, y establecen como variables tales como la edad, el género, los estudios, la formación, la experiencia previa y el trasfondo familiar, afectan a estos emprendedores que necesitan ayuda y apoyo de las incubadoras.

Al referirnos a la provincia de El Oro, se debe indicar que hay empresarios fuertemente posicionados en sectores productivos clave como el bananero y camaronero, con mucha 
experiencia en la comercialización internacional y con fuerte demanda de investigaciones específicas, sin olvidar otros sectores como el caficultor y cacaotero de larga tradición productiva, pero con poca absorción tecnológica.

Esto debe siempre estar muy bien identificado, ya que a veces pueden surgir situaciones en las que los facilitadores -especialmente las instituciones universitarias- intenten hacerse cargo de negocios en lugar de desempeñar su papel propio, que es apoyar el ecosistema empresarial, mediante investigación y desarrollo desde incubadoras tecnológicas.

Chandra, Chao, \& Astolpho (2014) al referirse a Brasil, manifiestan que una medida clave del éxito de una Incubadora de Negocios (IN) es su capacidad de vincular nuevos emprendimientos a los canales de los proveedores de conocimiento o recursos que pueden ser del gobierno, la industria o el mundo académico. Menciona además, que las incubadoras son en su mayoría organizaciones sin fines de lucro que operan en entornos con limitaciones de recursos, dependiendo de varias fuentes para su financiamiento operativo recurrente, tal como se lo mencionó anteriormente.

Por lo tanto, los vínculos con otras entidades organizacionales y empresariales ratifican la importancia de establecer alianzas para aliviar posibles déficits de recursos en las incubadoras que inician sus actividades.

Estos autores, indican que la universidad en Brasil ha tomado un rol directo en el impulso a la incubación, siendo las IES conectores entre los actores en la triple hélice (gobierno, las empresas y el mundo académico), además de proporcionar sus investigaciones de vanguardia y otros activos basados en el conocimiento, con lo que la línea de trabajo establecida en la octava economía mundial confirma la necesidad de trabajar en procesos de incubamiento.

Se reconocen además, que las investigaciones universitarias benefician a los nuevos emprendimientos alojados en una incubadora universitaria, con lo que se cumple con otro objetivo de la universidad como es la vinculación con la sociedad.

La naturaleza simbióticamente beneficiosa de la relación con todas las partes, potenciada por la ventaja local de la universidad en el nexo de interacción entre múltiples esferas, hace más probable que la incubadora universitaria tenga un mayor número de afiliaciones con respecto a otras incubadoras (Chandra et al., 2014).

En Ecuador existen de acuerdo a reportes de la Secretaría Nacional de Ciencia y Tecnología (SENESCYT) 80 incubadoras tanto públicas, privadas y mixtas, en lo público algunas son dependientes de universidades y otras de gobiernos autónomos descentralizados, con lo que el panorama es bastante amplio en torno al apoyo al emprendimiento.

De estas ochenta incubadoras, cuarenta han postulado para ser acreditadas por este organismo en el 2016, teniendo al final solo catorce seleccionadas entre las que existen solo tres incubadoras universitarias, con lo que el panorama de apoyo desde la universidad es limitado si se considera los estándares de calidad exigidos para acreditarse, demostrando que hay mucho 
trabajo por hacer desde la universidad pública para impulsar la generación de investigación y de innovación mediante propuestas empresariales.

Tabla 1. Incubadoras acreditadas por SENESCYT

\begin{tabular}{|c|c|c|c|c|c|c|c|c|c|}
\hline PROVINCIA & INCUBADORA & 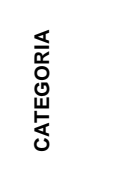 & 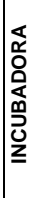 & 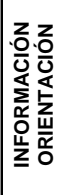 & 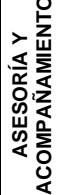 & 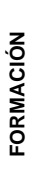 & 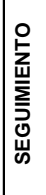 & 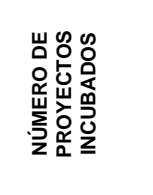 & 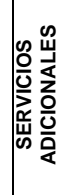 \\
\hline QUITO & AUNCONSIS CIA LTDA & PRIVADA & $\mathbf{x}$ & & $\mathrm{x}$ & & $\mathbf{x}$ & ENTRE 5 Y 10 & \\
\hline QUITO & CONQUITO & PRIVADA & $\mathbf{x}$ & $\mathrm{x}$ & $\mathrm{x}$ & & $\mathbf{x}$ & MAS DE 10 & \\
\hline LOJA & PRENDHO - UTPL & PRIVADA & $\mathbf{x}$ & $\mathrm{x}$ & $\mathrm{x}$ & $\mathrm{x}$ & $\mathrm{x}$ & MAS DE 10 & $\mathrm{x}$ \\
\hline QUITO & IMPAQTO & PRIVADA & $\mathbf{x}$ & $\mathbf{x}$ & $\mathbf{x}$ & & $\mathbf{x}$ & MAS DE 10 & \\
\hline QUITO & KRUGERLABS & PRIVADA & $\mathrm{x}$ & $\mathrm{x}$ & $\mathrm{x}$ & & $\mathrm{x}$ & MAS DE 10 & \\
\hline QUITO & YACHAY EP & PUBLICA & $\mathbf{x}$ & $\mathbf{x}$ & $\mathrm{x}$ & & $\mathbf{x}$ & MAS DE 10 & \\
\hline GUAYAQUIL & ESPOL & PUBLICA & $\mathrm{x}$ & $\mathrm{x}$ & $\mathrm{x}$ & $\mathbf{x}$ & $\mathrm{x}$ & MAS DE 10 & \\
\hline QUITO & $\begin{array}{c}\text { INCUBADORA DE } \\
\text { EMPRESAS PICHINCHA } \\
\text { CAPEIPI }\end{array}$ & MIXTA & $\mathrm{x}$ & $\mathrm{x}$ & $\mathrm{x}$ & & $\mathrm{x}$ & MAS DE 10 & \\
\hline CUENCA & $\begin{array}{l}\text { UNIVERSIDAD } \\
\text { POLITECNICA } \\
\text { SALESIANA }\end{array}$ & PRIVADA & $\mathrm{x}$ & $\mathrm{x}$ & $\mathrm{x}$ & & $\mathrm{x}$ & MAS DE 10 & \\
\hline AMBATO & CORPOAMBATO & MIXTA & $\mathbf{x}$ & $\mathbf{x}$ & $\mathrm{x}$ & & $\mathrm{x}$ & MAS DE 10 & \\
\hline $\begin{array}{c}\text { SANTO } \\
\text { DOMINGO }\end{array}$ & $\begin{array}{l}\text { CENTRO DE } \\
\text { CAPACITACION Y } \\
\text { FORMACCION } \\
\text { PROVINCIAL }\end{array}$ & PUBLICA & $\mathrm{x}$ & $\mathrm{x}$ & $\mathrm{x}$ & & $\mathrm{x}$ & ENTRE 5 Y 10 & \\
\hline GUAYAQUIL & $\begin{array}{l}\text { INNOBIS (Startup } \\
\text { Lab S.A) }\end{array}$ & PRIVADA & $\mathrm{x}$ & $\mathrm{x}$ & $\mathrm{x}$ & & $\mathrm{x}$ & ENTRE 5 Y 10 & \\
\hline QUITO & WORKING UP S.A. & PRIVADA & $\mathbf{x}$ & $\mathbf{x}$ & $\mathrm{x}$ & & $\mathbf{x}$ & MAS DE 10 & \\
\hline
\end{tabular}

Fuente: Informe de incubadoras acreditadas por SENESCYT. Elaboración de autores.

Se puede apreciar que seis ciudades concentran los procesos de incubación en Ecuador, siendo la ciudad de Quito la que tiene la mayoría de ellas. Según la información proporcionada por las catorce incubadoras acreditadas sus servicios e infraestructura son limitados. Teniendo las universidades una muy buena posibilidad de desarrollar procesos más eficientes al contar con infraestructura física y recurso humano especializado para el apoyo al emprendimiento, aunque hay que considerar que los presupuestos no siempre tienen suficientes recursos asignados a la inversión e investigación.

\section{Conclusiones}

El crecimiento de Internet, la tendencia hacia la innovación abierta, la tecnología y el cambio hacia una economía del conocimiento están impulsando a la universidad ecuatoriana a la búsqueda de soluciones empresariales enfocadas a usuarios concretos. Esto unido al crecimiento del espíritu empresarial académico y el enfoque de fomento de empresas centradas en la 
tecnología, hacen pensar que las incubadoras de negocios son una herramienta potente para el desarrollo económico de los pueblos.

Como se ha evidenciado en este trabajo investigativo, el éxito de una incubadora de empresas universitaria debe tomar en consideración la relación sinérgica entre los tres actores clave como son universidad, empresas y sociedad, sin dejar de lado el papel activo del gobierno central y seccional en el apoyo de la investigación empresarial y el desarrollo de nuevas iniciativas.

En este sentido, es necesario que para querer implementar una incubadora en una universidad, se deban contar con requisitos mínimos como los que se mencionaron anteriormente, como es contar con estudios de factibilidad y alianzas público privadas facilitadas por el marco normativo actual en Ecuador que permitan la comercialización de investigación universitaria.

Esta investigación también ha demostrado que las incubadoras de empresas son generadoras de desarrollo económico en países que las orientan a producir empresas de base tecnológica.

La presencia de incubadoras universitarias puede ayudar a que sus egresados puedan crear sus propios empleos, o convertirse en empresarios privados que ayuden a mejorar las condiciones productivas de los territorios en los que viven. Así como a la creación de redes universitarias y empresariales que desempeñen un papel fundamental en el fomento del espíritu empresarial académico y la creación de nuevas empresas de tecnología para establecer vínculos con la tecnología regional, recursos y el conocimiento.

En suma, este artículo trata de contribuir de manera importante al debate sobre el papel de las incubadoras de ciencia y tecnología en el crecimiento de las universidades, empresas y el desarrollo regional.

\section{Bibliografía}

Aerts, K., Matthyssens, P., \& Vandenbempt, K. (2007). Critical role and screening practices of

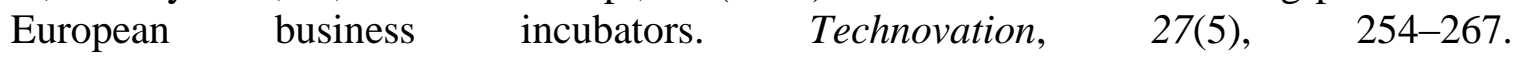
http://doi.org/10.1016/j.technovation.2006.12.002

Albert, P., Gaynor, L., 2001. Incubators: growing up, moving out - a review of the literature, Cahiers de Recherche, Arpent.

Albort-Morant, G., \& Oghazi, P. (2016). How useful are incubators for new entrepreneurs? Journal of Business Research, 69, 2125-2129. http://doi.org/10.1016/j.jbusres.2015.12.019

Arnaut, D. (2010). Towards an Entrepreneurial University. International Journal of EuroMediterranean Studies, 3(1). 
Asamblea Nacional del Ecuador. (2015). Ley orgánica de incentivos para asociaciones público privadas. Quito.

Bikse, V., Lusena-Ezera, I., Rivza, B., \& Volkova, T. (2016). The Transformation of Traditional Universities into Entrepreneurial Universities to Ensure Sustainable Higher Education. Journal of Teacher Education for Sustainability, 18(2), 75-88. http://doi.org/10.1515/jtes2016-0016

Chandra, A., Chao, C. A., \& Astolpho, E. C. (2014). Business incubators in Brazil: does affiliation matter? International Journal of Entrepreneurship and Small Business, 23(4), 436. http://doi.org/10.1504/IJESB.2014.065678

Chandra, A., He, W., \& Fealey, T. (2007). Business Incubators in China: A Financial Services Perspective. Asia Pacific Business Review, 13(1), 79-94. http://doi.org/10.1080/13602380601030647

Burton R. Clark (2003) Sustaining change in universities: Continuities in case studies and concepts, Tertiary Education and Management, 9:2, 99-116, DOI: 10.1080/13583883.2003.9967096

Consejo de Educación Superior. (2010). Ley Orgánica de Educación Superior, LOES.

Etzkowitz, H. (2003). Innovation in innovation: the Triple Helix of university-industrygovernment relations. Social Science Information Sur Les Sciences Sociales, 42, 293-337. http://doi.org/10.1177/05390184030423002

Etzkowitz, H., 2004. The evolution of the entrepreneurial university. International Journal of Technology and Globalisation 1 (1): 64-77

Freire, M. T. (2016). Educación en emprendimiento. Revisión de la literatura económica. Retrieved May 18, 2016, from http://revistas.ucm.es/index.php/IJHE/article/viewFile/52052/48101

Gibb, A., G. Haskins, and I. Robertson. 2009. Leading the entre- preneurial university: Meeting the entrepreneurial development needs of higher education institutions. Http://www.ncge.org.uk/ publication/leading_the_entrepreneurial_university.pdf.

Hannon, P. D. 2008. Entrepreneurship education strategies and best practice: Views, thoughts, challenges, opportunities. Http://ec. europa.eu/education/highereducation/doc/business/october08/hannon_en.pdf

Gozali, L., Masrom, M., \& Zagloel, T. (2016). A framework of successful business incubators for Indonesian public universities. International Journal of Technology, 1086-1096. 
Grimaldi, R., Kenney, M., Siegel, D. S., \& Wright, M. (2011). 30 years after Bayh-Dole: Reassessing academic entrepreneurship. Research Policy, 40(8), 1045-1057. http://doi.org/10.1016/j.respol.2011.04.005

Hernández, C., \& González, D. (2016). Study of the Start-Up Ecosystem in Lima, Peru: Collective Case Study. Latin American Business Review, 17(2), 115-137. http://doi.org/10.1080/10978526.2016.1171678

Peña-Vinces, J. C., Bravo, S., Álvarez, F. A., \& Pineda, D. A. (2011). Análisis de las características de las incubadoras de empresas en Colombia: un estudio de casos. Journal of Economics, Finance and Administrative Science, 16(30), 13-29.

Robertson, I. 2008. Comment: How universities and graduates can thrive. Http://www.independent.co.uk/student/career-planning/ getting-job/comment-howuniversities-and-graduates-can -thrive-918572.html

Smith, D. J., \& Zhang, M. (2012). Introduction: the evolution of the incubator concept. The International Journal of Entrepreneurship and Innovation, 13(4), 227-234. http://doi.org/10.5367/ijei.2012.0096

Theodorakopoulos, N., Kakabadse, N. K., \& Mcgowan, C. (2014). What matters in business incubation? A literature review and a suggestion for situated theorising. Journal of Small Business and Enterprise Development, 21(4). http://doi.org/10.1108/JSBED-09-20140152

Trippl, M., Sinozic, T., \& Lawton Smith, H. (2015). The Role of Universities in Regional Development: Conceptual Models and Policy Institutions in the UK, Sweden and Austria. $\begin{array}{llll}\text { European } \quad \text { Planning } & \text { Studies, } & \text { 23(9), }\end{array}$ http://doi.org/10.1080/09654313.2015.1052782

Verma, S. (2004). Success factors for business incubators an empirical study of canadian business incubators. Carleton University.

Wonglimpiyarat, J. (2016). The innovation incubator, University business incubator and technology transfer strategy: The case of Thailand. Technology in Society, 46, 18-27. http://doi.org/10.1016/j.techsoc.2016.04.002 